Submitted to IJAA: Rev \#1 21.08.18

\title{
The Interfacial Interaction between Isocyanate and Stainless Steel
}

\author{
Sabrina Tardio ${ }^{1+}$, Marie-Laure Abel ${ }^{1}$, Robert H Carr ${ }^{2}$, John F Watts ${ }^{1^{*}}$ \\ ${ }^{1}$ The Surface Analysis Laboratory' Department of Mechanical Engineering Sciences, \\ University of Surrey Guildford, Surrey, GU2 7XH, UK \\ ${ }^{2}$ Huntsman PU \\ Everslaan 4, B-3078 Everberg, Belgium \\ *j.watts@surrey.ac.uk
}

\begin{abstract}
The interfacial interactions between methylene diphenyl di-isocyanate (MDI) and polymeric MDI (PMDI) with 316L stainless steel have been studied in order to understand the adhesion properties of polyurethane based adhesives (containing free isocyanate groups) on metal surfaces. A thin ( $<5 \mathrm{~nm}$ ) layer of MDI and PMDI was deposited on the surface of clean $316 \mathrm{~L}$ stainless steel and then analysed by X-ray photoelectron spectroscopy (XPS) and time of flight secondary ion mass spectrometry (ToF-SIMS). By using density functional theory (DFT) methods for the interpretation of XPS data, the presence of specific interactions between the adsorbate and the substrate was established. The reaction between isocyanate and metal hydroxyl groups with the formation of urethane-like bonding with the metal was observed. The formation of hydrogen bonds between the isocyanate nitrogen and the hydroxyl groups and the formation of nitrogen-metal double bond, as consequence of the cycloaddition reaction between isocyanate and metal oxide, are also proposed.
\end{abstract}

Keywords: XPS, ToF-SIMS, polyurethanes, adsorption isotherm, polymer-metal interactions

$\dagger$ Current address: School of Mechanical \& Systems Engineering, Newcastle University, Newcastle upon Tyne NE1 7RU 


\section{Introduction}

Polyurethanes (PUs) are very commonly used adhesives and they tend to display strong adhesion to the surfaces of metal oxides. Strong adhesion is often caused by the formation of chemical bonds between the molecule of the adherent and those of the substrate [1]. Thus, it is important to study the interactions occurring at the relevant interfaces.

Polyurethanes are made of chains containing urethane bonding. These are obtained by reacting an isocyanates with hydroxyl groups. The great majority of di-isocyanate used in the polyaddition reaction is methylene diphenyl di-isocyanate (MDI) [2]. This can be considered as the PU monomer. Isocyanates have a very rich chemistry and some of the more important reactions involving $\mathrm{MDI}$ are shown in Figure 1.

Some adhesive formulations are based on PU monomers, and some pre-polymers contain unreacted isocyanate groups. This work aims to study the interaction of MDI and polymeric MDI (PMDI) with stainless steel surfaces. The importance of the MDI reactivity shown in Figure 1 can be easily understood considering that the steel surface contains hydroxide and adsorbed water [3]. It would be expected that the MDI can interact with the moisture from atmosphere and with those groups, forming urethane type of bond with the metal hydroxide and urea respectively. It was chosen to not perform the reaction under an inert gas to avoid the reaction with the air moisture since most industrial processes take place in atmosphere and it is important to take that in account when studying adhesion properties. Specific interactions were identified from the XPS and SIMS signals of the constituent elements of stainless steel and that of MDI. By constructing an adsorption isotherm and calculating the thickness of the chemisorbed layer, it was possible to estimate the geometry of interaction of MDI on steel, in the manner described by Watts and Castle [4].

\section{Experimental and Methods}

\section{Sample preparation}

Reference samples were prepared in the following way: MDI, PMDI, and urea were provided by Huntsman PU. Methylene diphenyl diamine (MDA) and the MDI dimer were provided by 
Sigma Aldrich (UK). Two samples of $10 \mathrm{~mm}^{2}$ were cut from aluminium sheets (provided by Goodfellow, UK) and covered with a thick layer of MDI and PMDI by drop coating in order to analyse the bulk of the compounds. The MDI dimer was analysed as compressed powder in an aluminium container. Pellets of the solid were used for MDA analysis. For urea, spectra acquired by Shimizu [5] were employed as a reference.

Samples of $10 \mathrm{~mm}^{2}$ were cut from 316L stainless steel sheets, provided by Goodfellow, UK. These coupons were successively etched free of the oxide in a Theta Probe (Thermo Scientific, East Grinstead, UK) XPS spectrometer. Ion etching to remove the pre-existing oxide was performed using a Thermo EX05 argon ion gun ( $3 \mathrm{keV} \mathrm{Ar}^{+}$, rastered over an area of $2.5 \times 2.5$ $\mathrm{mm}^{2}$ ), and was continued until a clean metal surface, free from oxide and adventitious carbon was revealed in the XPS survey spectrum.

The coupons were then exposed to laboratory air for a short time (30 minutes) in order to obtain a native air-formed oxide on the surface. Pure MDI and PMDI, as received, were diluted in acetone $(99.9 \%$ purity, water $\leq 0.5 \%)$ in order to obtain solutions in the range $10^{-3}$ $\mathrm{v} / \mathrm{v} \%$ to $10 \mathrm{v} / \mathrm{v} \%$. The $10 \mathrm{v} / \mathrm{v} \%$ was prepared by mixing $5 \mathrm{ml}$ of MDI or PMDI with acetone in a $50 \mathrm{ml}$ volumetric flask. The other solutions were prepared by successive dilutions. The steel coupons were immersed in $40 \mathrm{~cm}^{3}$ of the chosen solution for 20 minutes to establish kinetic equilibrium [5]. No further polymerization was performed. The samples were then rinsed with fresh acetone three times for two minutes and left to drain in a vertical orientation on aluminium foil and then left to dry for at least 1 hour. These specimens were then analysed by XPS and the data used to construct the adsorption isotherm of MDI on steel.

\section{XPS Analysis}

All the samples prepared were analysed by XPS. The survey spectra were acquired in the constant analyser mode at pass energy of $300 \mathrm{eV}$ with a step size of $1 \mathrm{eV}$ (in order to avoid potential damage, to the sample), the high resolution spectra at a pass energy of $30 \mathrm{eV}$ with step size of $0.1 \mathrm{eV}$. A monochromated Al K $\alpha$ X-ray beam was employed, with a spot size of $400 \mu \mathrm{m}$. Spectral acquisition and subsequent processing was carried out using the instrument manufacturer software (Avantage V5.938).

The Avantage data system provided the sensitivity factors and transmission function correction required for the calculation of a quantitative surface analysis. Peak areas of the 
high resolution spectra were employed following the removal of a Shirley (S-type) background. For all the peak fitting, a 30\% Voigt function was used. In the first instance, the full width at half maximum (FWHM) was kept the same for the different components of a given photoelectron peak, however it was allowed to vary slightly from this position on a second iteration. As a general criterion, FWHM were kept the same for components of the same photoelectron peaks, while, for different photoelectron peaks, increasing FWHM were selected with the increase of the binding energy. Data from literature were employed as a reference for peak positions [6], with a small constraint applied for peak fitting.

\section{DFT Binding Energy Simulation}

In this work a Koopmans' theorem based approach was employed, combined with a tuning procedure which improves the binding energy predictions to a level comparable with more complex calculations [7]. The binding energies of the electrons of interest were calculated by using the functional B3LYP [8] and the basis set 6-311G(d,p) and performed with the GAUSSIAN 09 program package. The first step was to optimize the geometry of the molecules of interest. The second step was to employ the natural bond orbital (NBO) analysis to obtain the core orbital energies. This approach has recently been refined and extended to a number of homopolymers and is described in detail elsewhere [9].

\section{SIMS Analysis}

A TOF.SIMS 5 (ION-TOF GmbH, Münster, Germany) system was employed for ToF-SIMS analyses. Static SIMS conditions (ion dose $<10^{13}$ ions $\mathrm{cm}^{-2}$ ) were employed using a $25 \mathrm{keV} \mathrm{Bi}_{3}{ }^{+}$ primary ion beam, with $9.5 \mathrm{keV}$ extractor voltage, rastered over an area of $100 \times 100 \mu \mathrm{m}^{2}$. Both positive and negative SIMS spectra were acquired in the high spectral resolution (high current bunched mode) mode over a mass range of $1-850 \mathrm{u}$. Spectral acquisition and processing was achieved using ION-TOF GmbH SurfaceLab v 6.4 software. The accuracy of the mass assignment $(\Delta)$ was calculated as expressed in Equation 1.

$$
\Delta=\frac{\left|M_{e x}-M_{r}\right|}{M_{r}} \cdot 10^{6}
$$


Where $\Delta$ in the accuracy of the mass assignment in $\mathrm{ppm}, \mathrm{M}_{\mathrm{ex}}$ is the experimentally measured mass in the spectra and $M_{r}$ is the exact (real) mass of the candidate fragment. Calibration of the spectra was obtained by assigning at least 10 known peaks spread throughout the mass range considered. All the assignments made had a $\Delta<100 \mathrm{ppm}$, indicating the assignment is valid.

\section{Results and discussion \\ DFT method tuning procedure}

The reference samples; MDI, MDI Dimer (which can be formed from MDI interacting with itself), MDA and Urea (formed when MDI reacts with water) were analysed by XPS and peak fitting routine of the $\mathrm{C} 1 \mathrm{~s}$ feature was attempted. Theoretical $\mathrm{C} 1 \mathrm{~s}$ binding energies of the reference molecule were obtained by DFT. The values obtained were compared to those obtained by XPS. By plotting the binding energies of the C1s functional groups obtained with the calculation against the one obtained by XPS, a pretty good fit of a line is obtained [10], the $R^{2}$ value of the linear regression which fit the data is 0.94 . From the equation of the line obtained, the following tuning procedure, to improve the binding energy prediction, is implemented (Equation 2).

$$
\left.E_{C 1 s}^{\text {exp }}=0.9184 E_{C 1 s}^{\text {theory }}+33.648 \quad(e V) \quad 2\right)
$$

where $\mathrm{E}_{\mathrm{C} 1 \mathrm{~s}}{ }^{\text {exp }}$ is the experimental or correct $\mathrm{C} 1 \mathrm{~s}$ binding energy, and $\mathrm{E}_{\mathrm{C} 1 \mathrm{~s}}{ }^{\text {theory }}$ is $\mathrm{C} 1 \mathrm{~s}$ binding energy calculated by DFT. The binding energies obtained using this tuning procedure and the binding energies obtained by XPS are shown in Table 1.

The theoretical values agree well with the experimental values (with a standard deviation of $0.4 \mathrm{eV}$ ) if compared to more complex and accurate DFT binding energies estimations. Also, the same number of peaks observed experimentally can be predicted on the basis of the binding energies calculated. This method will successively be used to help the understanding and interpretation of the interface spectra and support the suggested assignments. For the 
$\mathrm{N} 1 \mathrm{~s}$ chemical shift, there is not sufficient data to build a regression line, in the manner of C1s above. Thus, a line made by using theoretical and experimental binding energies of C1s, N1s and $\mathrm{O} 1 \mathrm{~s}$ data together was built. The tuning procedure, which can be employed for N1s binding energies prediction, is shown in Equation 3. In this case the standard deviation is 0.6 eV.

$$
\left.E_{N 1 s}^{\text {exp }}=1.0274 E_{N 1 s}^{\text {theory }}+3.623(e V) \quad 3\right)
$$

\section{Geometry of interaction}

The samples made by exposing the steel coupons to the MDI solutions in acetone for 20 minutes in the range $10^{-3} \mathrm{v} / \mathrm{v} \%$ to $10 \mathrm{v} / \mathrm{v} \%$ were used to build the adsorption isotherm of MDI on steel.

Since the nitrogen is indicative of the presence of the MDI on the steel, its concentration was considered to be proportional to the MDI solution concentration in order to obtain adsorption isotherms in the manner of Watts and Castle [4]. To determine whether monolayer coverage for the higher concentrations was observable, the Langmuir test was carried out [4] and the isotherm was confirmed to be of Langmuir type. The MDI overlayer thickness for the sample obtained at the plateau of the isotherm, calculated by means of the Beer-Lambert equation (Equation 4). This was achieved, as indicated below, by comparison of the intensity of the C1s signal from the candidate layer $\left(I_{d}\right)$ and a thick layer $>>1 \mu \mathrm{m}\left(I_{\infty}\right)$ applied to aluminium:

$$
\left.I_{d}=I_{\infty}(1-\exp [-d / \lambda \cos \theta]) \quad 4\right)
$$

Where the additional terms represent the electron attenuation length $(\lambda)$ and the electron take off angle relative to the sample normal $(\theta)$. This gives a result of MDI thickness on $316 \mathrm{~L}$ stainless steel of $1.1 \mathrm{~nm}$. The dimensions of the MDI molecule, obtained through DFT geometry optimization, are shown in Figure 2.

The measured thickness of the MDI layer $(1.1 \mathrm{~nm})$ is in the range of the molecule dimensions $(1.4,0.4$ and $0.4 \mathrm{~nm}$, in $\mathrm{x}, \mathrm{y}$ and $\mathrm{z}$ directions). Since the layer adsorbed is a monolayer (as confirmed by the Langmuir test), the comparison between the molecule dimensions and the overlayer thickness suggests that that the molecule is oriented on the surface with an angle of $50-60^{\circ}$ from the substrate surface, as shown in Figure 3. A similar method for the 
determination of the orientation of molecules on the surface have been used in the past [11]. This means that the interaction between adsorbate and substrate occurs with one end of the molecule.

A monodentate attack has been previously observed by Nies et al. in $[12,13]$ on other metals. A reaction between the isocyanate group and the hydroxide, present on the surface of the steel, can thus be supported. As it is possible to see from Figure 3, the formation of this covalent bond is compatible with the possible orientation of the molecule on the surface.

\section{XPS Results}

The survey spectra for $316 \mathrm{~L}$ steel surface, (following sputter cleaning in the XPS spectrometer and subsequent exposure to air for 30 minutes), MDI, PMDI and of the MDI and PMDI exposed $316 \mathrm{~L}$ steel coupons are shown in Figure 4. The spectra of the steel exposed to the organic molecules look like hybrids between the spectra of the oxidised metal surface and pure molecules. The presence of both nitrogen and substrate peaks ( $\mathrm{Fe}$ and $\mathrm{Cr}$ ) in the spectra confirms that the interface between the two materials is the dominant contribution to the region analysed, and will be referred to as the interfacial region in descriptions of spectra in subsequent figures. In Figure 5, high resolution C1s spectra of pure MDI and PMDI and of their interfacial regions with stainless steel are shown. The spectra have been fitted and the different components have been assigned. The MDI bulk (which looks the same as the PMDI bulk) spectra show a C-C, C-H component due to the aromatic ring and the methyl group, a component caused by the $\mathrm{C}-\mathrm{N}$ bonding (between the carbon of the ring and the nitrogen of the isocyanate group) a peak due to the isocyanate $(\mathrm{N}=\mathrm{C}=\mathrm{O})$, and the shake-up feature of the aromatic ring.

The interface sample C1s spectra exhibits features of both MDI (or PMDI) and steel; in addition to the peaks seen on the pure samples, a $\mathrm{C}-\mathrm{O}-\mathrm{C}=\mathrm{O}$ peak and more prominent $\mathrm{C}-\mathrm{C}=\mathrm{O}$ and $\mathrm{C}-\mathrm{O}$ features, caused by the presence of carbon contamination on the surface of the steel, are present. A reduction of the shake-up intensity is also observed. Detailed spectra of the $\mathrm{N}=\mathrm{C}=\mathrm{O}$ regions for pure $\mathrm{MDI}$ and the two interfacial samples are shown in Figure 6 . The inspection of these spectra shows that the NCO peaks of the interfacial region samples are wider (with a FWHM = $1.4 \mathrm{eV}$ if fitted with one peak) compared to the other components of 
the C1s $(F W H M=1.1)$ and compared to the NCO peak of the reference sample (FWHM $=1.0$ $\mathrm{eV})$. The features are also asymmetric suggesting the presence of two peaks under the curve. This, hence, hints at the presence of particular species at the interface. This peak can be fitted with two components which may be assigned to $\mathrm{N}=\mathrm{C}=\mathrm{O} / \mathrm{R}-\mathrm{C}=\mathrm{O}$ and $\mathrm{HNCOOM}(\mathrm{M}=$ metal), the shake-up signal is visible for the MDI interface at higher binding energies, but is almost not visible on the PMDI interface spectrum. It is possible that because of the higher steric hindrance of PMDI, not as many isocyanate group are able to interact with the surface of steel, compared with pure MDI. This results in less aromatic structures being present on the surface and, consequently, a weaker satellite structure.

Since the suggested interaction involves the nitrogen, matching information should be sought in the N1s spectra. Figure 7 shows the N1s high resolution spectra of MDI and the two interfacial samples. The nitrogen peak of MDI, as expected, shows only one peak; that of the isocyanate. The interface samples, by contrast, are made by three components. The peak at higher binding energy than isocyanate is compatible with the formation of urethane type of bonding [6] (thus matching the observation made on the C1s peak), and also with the formation of hydrogen bonding between the nitrogen and the metal hydroxide [14]. The third component, at a lower binding energy than the N1s of the isocyanate group, can be attributed to an interaction of the nitrogen with the metal [15]. A broadening is also observed on both the lower (around $399 \mathrm{eV}$ ) and higher (around $401 \mathrm{eV}$ ) binding energy side of Figure 7(b) and the lower (around $399 \mathrm{eV}$ ) binding energy side of Figure 7(a). These features are likely not of spectroscopic significance (attempts to peak fit them did not provide sensible solutions), but are merely a result of the inherently poor counting statistics of the N1s spectra, as the nitrogen is at a rather low concentration for these two samples. This is indicated by the XPS survey spectra of Figure 5 (lower two spectra in orange and blue).

The oxygen signal, containing peaks originating from both the substrate and the adsorbed layer is too complex to give information on the interface; this is also true because the chemical shifts observed on 01s are smaller than those observed on C1s. No differences between the Fe2p and $\mathrm{Cr} 2 \mathrm{p}$ signals of the interface samples and of oxidised steel are observed.

XPS analysis seems then to suggest three types of interactions; one involving the reaction of the isocyanate with the metal hydroxide and two involving the reaction between the nitrogen of the MDI and metal oxide and hydroxide. For the first interaction, the mechanism of 
reaction, which is the same occurring for the formation of the urethane bonding [2], is shown schematically in Figure 8.

The hydroxide oxygen doublet is attracted by the electrophilic carbon of the isocyanate group, this will leave the nucleophilic nitrogen free to interact with the hydrogen in a four centres reaction mechanism.

Two interactions involving the nitrogen can be observed; the nitrogen can adsorb on the metal through hydrogen bonding with the metal hydroxide and can also form a covalent bond between nitrogen and the metal. This can be due to a cycloaddition reaction between the isocyanate group and the metal oxide [16] (see Figure 9).

The middle step of this mechanism provides the same type of bonding to that obtained through the interaction between isocyanate and hydroxide. This means that this type of mechanism can also contribute to the formation of the first type of covalent bond with the metal.

\section{DFT calculation}

In order to confirm the assignments, DFT prediction for the binding energy of C1s and N1s in the product molecules was employed. The molecules used to simulate the products created are illustrated in Figure 10.

The theoretical binding energies for the possible products of interaction are shown in Table 2 where they are compared with the experimental binding energies found by XPS in the interface. In order to have a reference, theoretical and experimental binding energies for pure MDI have also been added.

As it is possible to see, the theoretical data, if not fully confirming, at least support the assignments made for the new XPS peaks appearing at the interface. In the case of C1s the predicted binding energies values for the formation of the suggested products of reaction are very close to the experimental ones. In the case of the N1s, although the values are not as close, the trend of the theoretical and experimental results is the same; also, it needs to be considered that the approximate method used for the DFT calculation is less accurate on the N1s than the C1s (since less reference data were available as mentioned previously). Based on the DFT calculation, higher binding energies (compared to the isocyanate group) are 
expected for both C1s and N1s as a consequence of the formation of the urethane type of bond with the metal. A lower N1s binding energy is instead expected for the product of the cycloaddition reaction of isocyanate to metal oxide. All these characteristics are observed in the XPS spectra of the interfacial samples.

These results are in general agreement with works available in the literature. Chehimi and Watts [17], through the C1s peak of a thin layer of PU resin on steel, showed that the species involved in the interfacial interaction are the oxygenated and nitrogenated groups with double bonds. Dillingham and Moriarty [18] proposed the reaction of isocyanate with water adsorbed on the surface of the steel with the formation of carbamic acid which could react with isocyanate to form urea (which was not detected) but prefers to react with metal hydroxide to form carboxylate anion associated with protonated metal hydroxide.

Similar results have also been reported on aluminium surfaces; Kim et al. [19] suggested that a strong interaction between isocyanate and aluminium hydroxide can be expected which was confirmed by Shimizu et al. [20].

\section{ToF-SIMS Results}

In parallel with XPS analysis, MDI, PMDI, urea and the interface samples were also analysed by ToF-SIMS. The SIMS positive spectra of pure PMDI and PMDI-steel interface are shown in Figure 11. The assignment of the most important fragments is given in Figure 12. It is interesting to observe how the fragments containing isocyanate $(\mathrm{m} / \mathrm{z}=132,206$ and $249 \mathrm{u})$ are more intense in the bulk of the PMDI while at the interface the ions containing amine $(\mathrm{m} / \mathrm{z}=106,195$, and $197 \mathrm{u})$ become more intense. This means that there is loss of CO at the interface indicating reactions occurring.

The data show how nitrogen containing fragments are more important at the interface than in the original molecule. An increase in intensity of these peaks corresponds to a decrease in intensity of the peaks from hydrocarbon fragments and oxygen containing fragments. This is represented in Figure 13 which illustrates the high resolution SIMS spectra of the fragments at nominal mass of $28 \mathrm{u}$ for pure PMDI and PMDI at the interface. This result has two possible explanations. One is that the isocyanate interacts with water forming urea with loss of $\mathrm{CO}_{2}$ (see Figure 1 (b) and (c)) increasing thus the nitrogen carbon ration. The second is that, as observed by XPS, the formation of hydrogen bonding between the nitrogen of the isocyanate 
and the hydroxide of the metal leads to the nitrogen being more retained on the surface. Other important information can be obtained by identifying peaks which appear only in the interface samples and do not appear either in the substrate or pure organic molecule spectra. Some examples and relative fragment assignment are shown in Figure 14.

All these peaks originating from the interface have a mass lower than nominal mass which makes them likely to contain inorganic elements, in this case probably iron. The presence of peaks containing both metal and nitrogen confirms the formation of a covalent interaction between the steel and $\mathrm{MDI}$. The peaks assigned to $\mathrm{FeNH}_{2}{ }^{+}, \mathrm{C}_{6} \mathrm{H}_{6} \mathrm{NFe}^{+}$, and $\mathrm{CNOFe}^{+}$, respectively; $71.952 \mathrm{u}(\Delta=15: 6 \mathrm{ppm}), 147.985 \mathrm{u}(\Delta=23.1 \mathrm{ppm}), 97.931$ ( $\Delta=6.3 \mathrm{ppm})$ support the hypothesis of a cycloaddition reaction between metal oxide and isocyanate. This is because the fragments $\mathrm{FeNH}_{2}{ }^{+}$and $\mathrm{C}_{6} \mathrm{H}_{6} \mathrm{NFe}^{+}$are, in fact, likely to derive from the product of the reaction shown in Figure 9 while $\mathrm{CNOFe}^{+}$, could be formed from a rearrangement of the intermediate product of the mechanism of the same reaction. This last peak together with the peak at 117.952 assigned to $\mathrm{CH}_{4} \mathrm{NO}_{2} \mathrm{Fe}^{+}(\Delta=52.3 \mathrm{ppm})$ confirms the hypothesis of the formation of a urethane type bond with the metal through reaction of the isocyanate with the metal hydroxide. The fragment $\mathrm{CNOFe}^{+}$can also be obtained from a rearrangement of the product of the reaction shown in Figure 8 and $\mathrm{CH}_{4} \mathrm{NO}_{2} \mathrm{Fe}^{+}$is clearly a fragment of the reaction product. The same peaks are observed for both the MDI and PMDI interface samples.

\section{Conclusions}

The comparison between the thickness of the adsorbed monolayer and the dimension of the molecule (obtained by geometry optimization) suggests that MDI is adsorbed on to the surface with a pseudo-vertical orientation $\left(56-60^{\circ}\right)$ and, thus, that the bond with the substrate occurs with one end of the MDI molecule; the isocyanate functional group. Considering the isocyanate reactivity it is plausible that a reaction occurs between the isocyanate and the hydroxide present on the surface of the metal. This will lead to the formation of a covalent, urethane-like, bond between the molecule and the substrate. The formation of this bond was confirmed by XPS and ToF-SIMS analysis. Also other types of interactions could be detected; the formation of hydrogen bonding between nitrogen and metal hydroxide and formation of nitrogen metal double bond as result of a cycloaddition reaction between the isocyanate and 
the metal oxide. All these interactions between isocyanate and steel will lead to good adhesion of polyurethane based adhesive and metal oxide.

ORCID: John F Watts: https://orcid.org/0000-0003-2109-0335

\section{References}

(1) Kinloch A. J. Adhesion and Adhesives; Chapman and Hall, 1987.

(2) Randall D.; Lee S. The Polyurethanes Book; Wiley, 2001.

(3) Tardio S.; Abel M-L.; Carr R. H.; Castle J. E.; Watts J. F.; Comparative study of the native oxide on 316L stainless steel by XPS and ToF-SIMS: J. Vac. Sci. Technol. A. 2015, 33, 05E122-1-14.

(4) Watts J. F.; Castle J. E.; The determination of adsorption isotherms by XPS and ToF-SIMS: their role in adhesion science: Int. J. Adhes. Adhes. 1999, 19, 435-443.

(5) Shimizu K. The Interfacial Chemistry of Isocyanate/Metal Systems. Ph.D. Thesis, University of Surrey, Guildford, UK, 2011.

(6) Beamson G.; Briggs D.; High Resolution XPS of Organic Polymers: The Scienta ESCA300 database; Wiley, 1992.

(7) Chang D. P.; Accurate Density-Functional Calculation of Core-Electron Binding Energies of C1 to C4 Hydrocarbons: Chinese J. Phys. 2000, 38, 57-63.

(8) Becke D.; Density-functional thermochemistry. III. The role of exact exchange, J. Chem. Phys. 1993, 98, 5648-5652.

(9) Tardio S.; Cumpson P. J.; Practical Estimation of XPS Binding Energies using WidelyAvailable Quantum Chemistry Software, Surf. Interf. Anal., 2018, 50, 5-12.

(10) Giesbers M.; Marcelis A. T. M.; Zuilhof H.; Simulation of XPS C1s Spectra of Organic Monolayers by Quantum Chemical Methods: Langmuir. 2013, 29, 47824788.

(11) Taylor A. M., McLean C. H., Charlton M., Watts J. F.; Influence of Formulation Chemistry on the Locus of Failure of Adhesive Joints: Surf. Interf. Anal., 1995, 23, 342-348. 
(12) Nies C; Wehlack C.; Ehbing H.; Dijkstra D. J.; Possart W.; Adhesive Interactions of Polyurethane Monomers with Native Metal Surfaces: J. Adhes. 2012, 88, 665683.

(13) Fug F.; Nies C.; Possart W.; in situ FTIR study of adhesive interactions of 4,4'methylene diphenyl diisocyanate and native metals: Int. J. Adhes. Adhes. 2014, $52,66-76$.

(14) Biwer B. M.; Bernasek S. L.; Electron spectroscopic study of the iron surface and its interaction with oxygen and nitrogen: J. Electron Spectrosc. Relat. Phenom. 1986, 40, 339-351.

(15) XPS Knowledge Base, Thermo Fisher online database, http://xpssimplified.com.

(16) Ulrich H.; Chemistry and Technology of Isocyanates, Wiley, 1996.

(17) Chehimi M. M.; Watts J. F.; An XPS study of the steel-aromatic moisturecured urethane interface: J. Adhes. Sci. tech. 1992, 6, 337-393.

(18) Dillingham R. G.; Moriarty C.; The adhesion of isocyanate-based polymers to steel: J. Adhes. 2003, 79-269-285.

(19) Kim J.; Cho J.; Lim Y. S.; Bonding of urethane reactants to aluminum surface: J. Mater. Sci. 2005, 40, 2789-2794.

(20) Shimizu K.; Phanopoulos C.; Loenders R.; Abel M-L.; Watts J. F.; The characterization of the interfacial interaction between polymeric methylene diphenyl diisocyanate and aluminum: a ToF-SIMS and XPS study: Surf. Interface Anal. 2010, 42, 1432-1444. 


\section{Tables}

Table 1: Theoretical and experimental binding energies ( $\mathrm{BE} / \mathrm{eV})$ of the carbon functionalities of the reference compounds.

\begin{tabular}{|c|c|c|c|}
\hline Molecule & Functionality & XPS BE & DFT BE \\
\hline \multirow[t]{3}{*}{ MDI } & $\mathrm{C}-\mathrm{C} / \mathrm{C}-\mathrm{H}$ & 284.6 & 284.8 \\
\hline & $\mathrm{C}-\mathrm{N}$ & 285.6 & 286.3 \\
\hline & $\mathrm{N}=\mathrm{C}=\mathrm{O}$ & 288.9 & 2883 \\
\hline \multirow[t]{3}{*}{ MDI Dimer } & $\mathrm{C}-\mathrm{C} / \mathrm{C}-\mathrm{H}$ & 284.6 & 284.5 \\
\hline & $\mathrm{C}-\mathrm{N}$ & 285.7 & 286.2 \\
\hline & $\mathrm{N}_{2} \mathrm{CO}$ & 289.0 & 289.4 \\
\hline \multirow[t]{2}{*}{ MDA } & $\mathrm{C}-\mathrm{C} / \mathrm{C}-\mathrm{H}$ & 284.5 & 284.0 \\
\hline & $\mathrm{C}-\mathrm{N}$ & 285.5 & 285.3 \\
\hline \multirow[t]{3}{*}{ Urea } & $\mathrm{C}-\mathrm{C} / \mathrm{C}-\mathrm{H}$ & 284.6 & 284.5 \\
\hline & $\mathrm{C}-\mathrm{N}$ & 285.5 & 285.7 \\
\hline & $\mathrm{N}_{2} \mathrm{CO}$ & 289.1 & 288.5 \\
\hline
\end{tabular}

Table 2: Theoretical and experimental carbon functionalities binding energies.

\begin{tabular}{|l|l|l|l|}
\hline \multirow{4}{*}{ Molecule } & Functionality & XPS BE $(\mathrm{eV})$ & DFT BE $(\mathrm{eV})$ \\
\hline \multirow{5}{*}{ Ph-NHCOOFe } & C1s C-C/C-H & 284.6 & 284.8 \\
\cline { 2 - 4 } & C1s: C-N & 285.6 & 286.3 \\
\cline { 2 - 4 } & C1s: N=C=O & 288.8 & 288.3 \\
\cline { 2 - 4 } & N1s: N=C=O & 400.0 & 400.1 \\
\hline & C1s C-C/C-H & 284.8 & 284.4 \\
\cline { 2 - 4 } & C1s: C-N & 285.5 & 285.8 \\
\cline { 2 - 4 } & C1s: NCOOFe & 289.2 & 288.9 \\
\cline { 2 - 4 } & N1s: NCOOFe & 400.7 & 284.7 \\
\hline Ph-N=Fe & C1s C-C/C-H & 284.8 & 285.6 \\
\cline { 2 - 4 } & C1s: C-N & 285.5 & 399.7 \\
\cline { 2 - 4 } & N1s: N=Fe & 398.6 & \\
\hline
\end{tabular}




\section{Figures Captions}

Figure 1: Isocyanate reaction with: a) alcohol to give carbamate (urethane), b) water to give carbamic acid which breaks down to amine and carbon dioxide, c) amine to give urea, d) urea to give biuret and e) urethane to give allophanate.

Figure 2: 4-4' MDI optimized geometry shown on the $x-z$ and $y-z$ plane.

Figure 3: Geometry of the covalent bond formation at the interface between MDI and stainless steel.

Figure 4: XPS survey spectra of oxidised 316L steel (red), MDI (purple), PMDI (green), MDI316L interface (yellow) and PMDI-316L (blue).

Figure 5: C1s high resolution spectra of a) oxidised 316L steel, b) MDI bulk, c) MDI-316L interface and d) PMDI 316L interface.

Figure 6: XPS C1s NCO region high resolution spectra of a) MDI-316L interface, b) PMDI-316L interface and c) MDI bulk.

Figure 7: XPS N1s high resolution spectra of a) MDI-316L interface, b) PMDI-316L interface and c) MDI bulk.

Figure 8: Schematic representation of the possible mechanism for the reaction of MDI with iron hydroxide.

Figure 9: Schematic representation of the possible mechanism for the reaction between nitrogen in MDI and iron oxide.

Figure 10: Model molecules used for DFT binding energy prediction for the product of a) reaction between $\mathrm{MDI}$ and iron hydroxide and $\mathrm{b}$ ) cycloaddition reaction of MDI to iron oxide.

Figure 11: SIMS positive spectrum from 10 to 250 mass (u) of a) PMDI, b) PMDI-316L interface.

Figure 12: Possible structure of positive ion fragments from PMDI and relates molecules.

Figure 13: Pure PMDI and PMDI-316L interface peaks at $28 \mathrm{u}$.

Figure 14: SIMS positive peaks around a) $72 \mathrm{u}$, b) $98 \mathrm{u}$, c) $118 \mathrm{u}$ and d) $148 \mathrm{u}$ nominal mass. 
a)

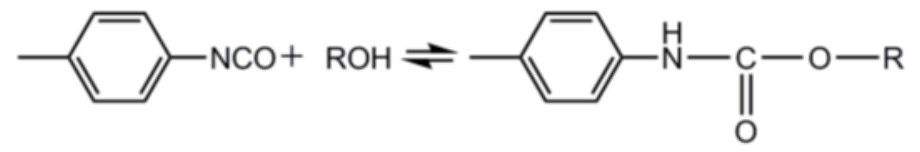

b)

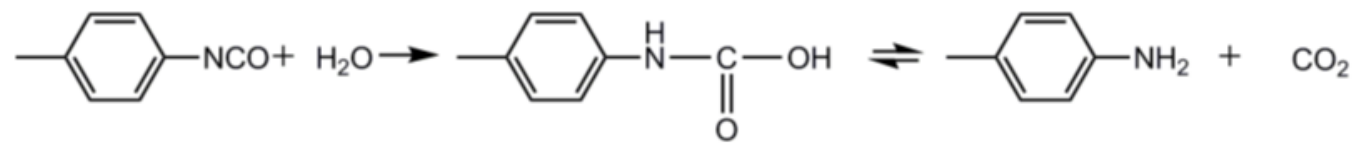

c)

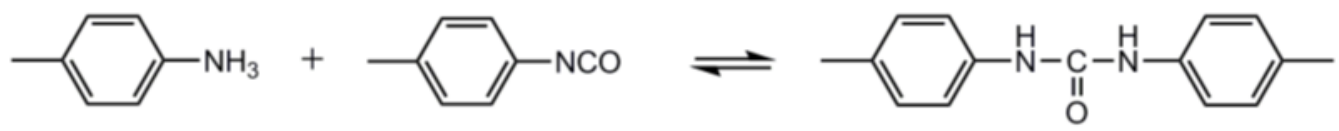

d)

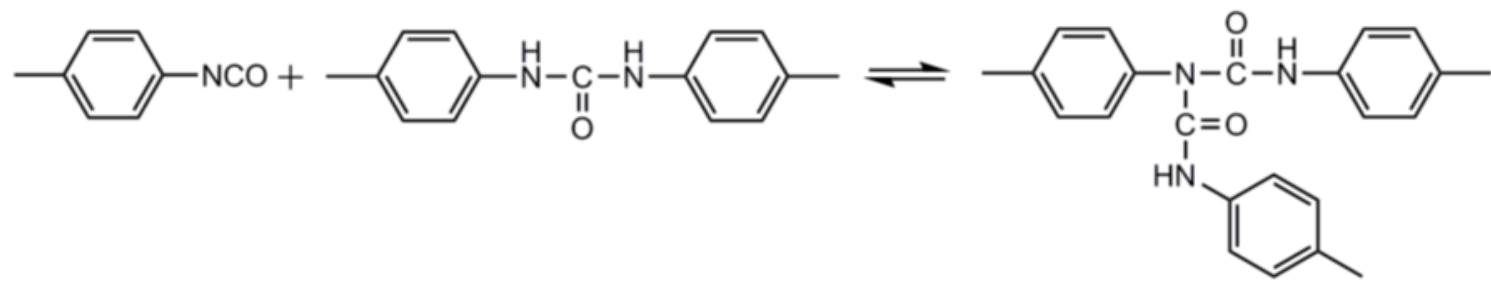

d)
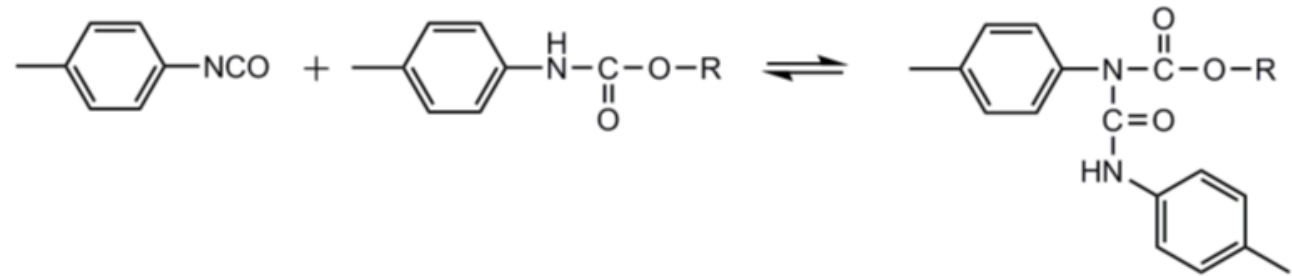

Figure 1: Isocyanate reaction with: a) alcohol to give carbamate (urethane), b) water to give carbamic acid which breaks down to amine and carbon dioxide, c) amine to give urea, d) urea to give biuret and e) urethane to give allophanate.

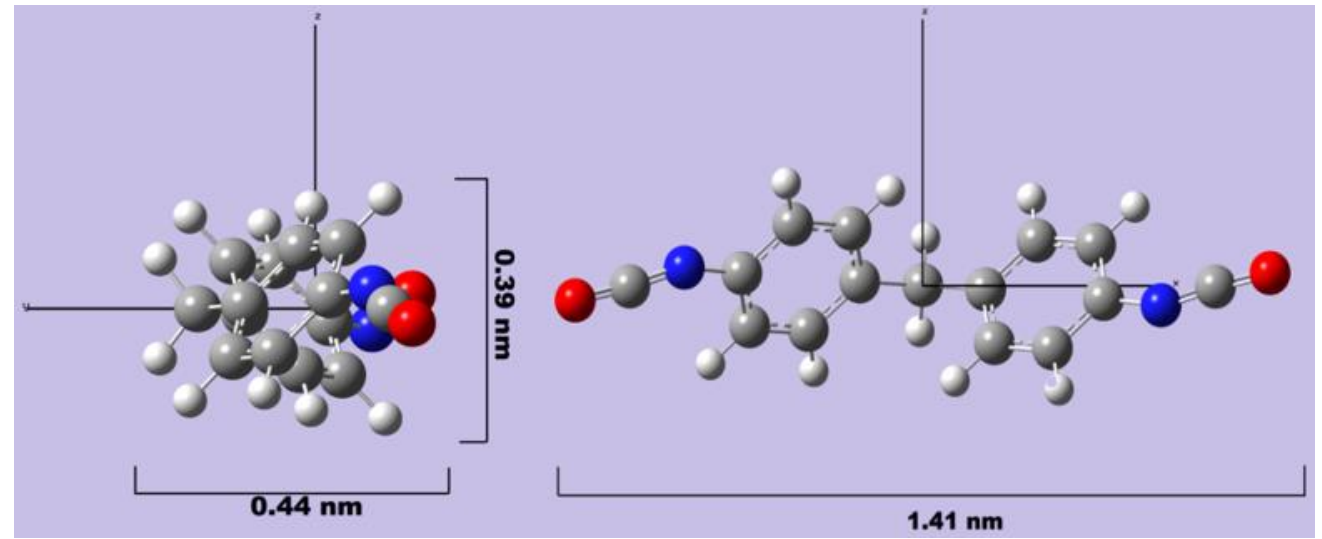

Figure 2: 4-4' MDI optimized geometry shown on the $x-z$ and $y-z$ plane. 


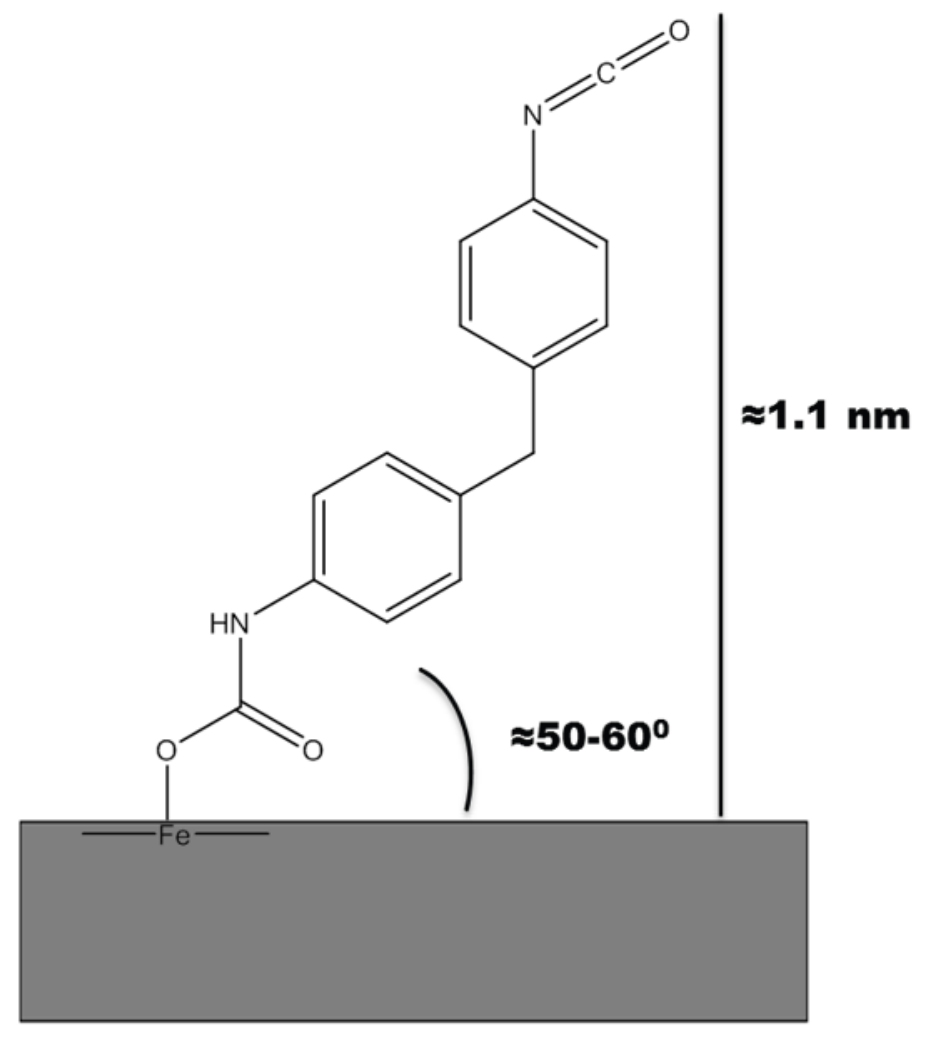

Figure 3: Geometry of the covalent bond formation at the interface between MDI and stainless steel. 


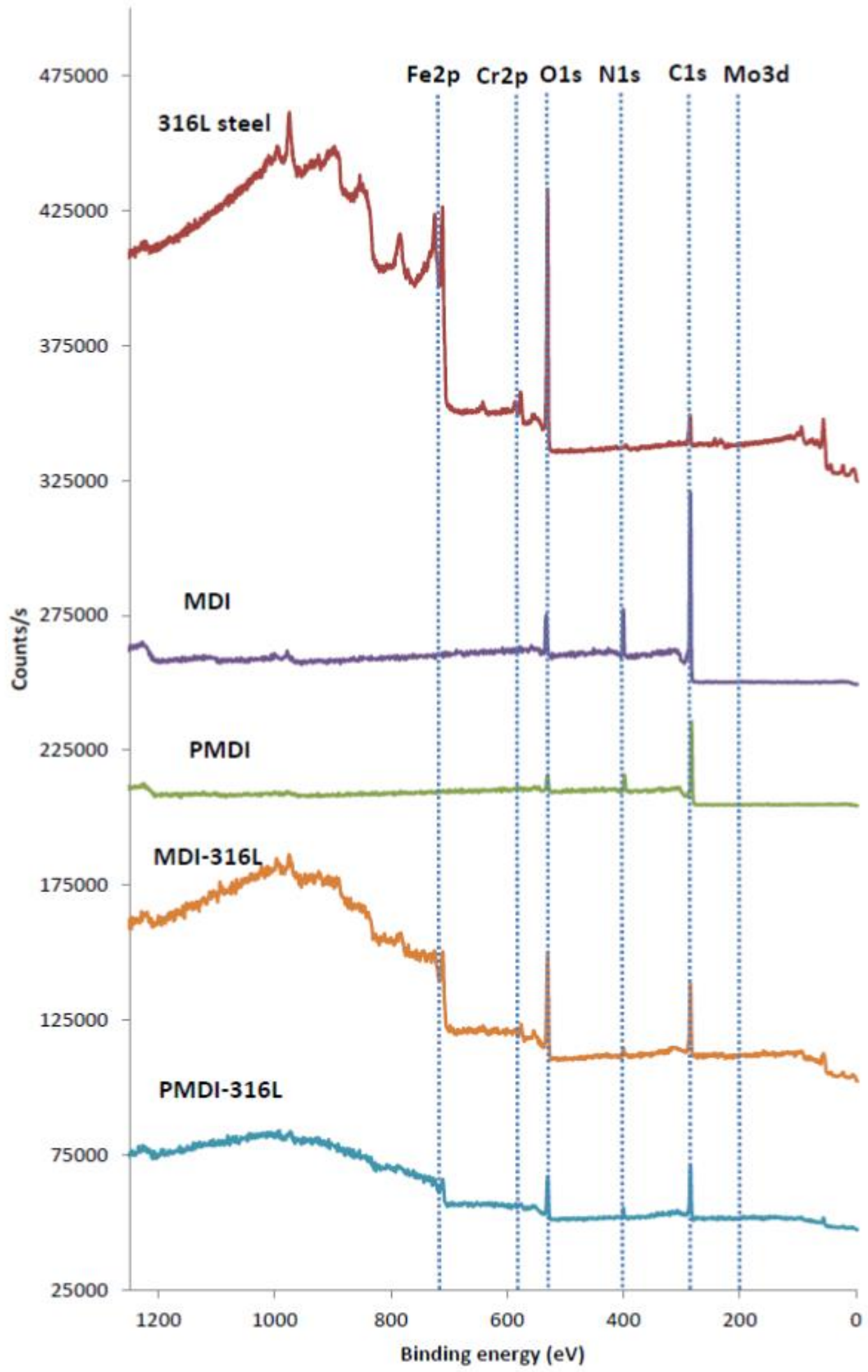

Figure 4: XPS survey spectra of oxidised 316L steel (red), MDI (purple), PMDI (green), MDI316L interface (yellow) and PMDI-316L (blue). 

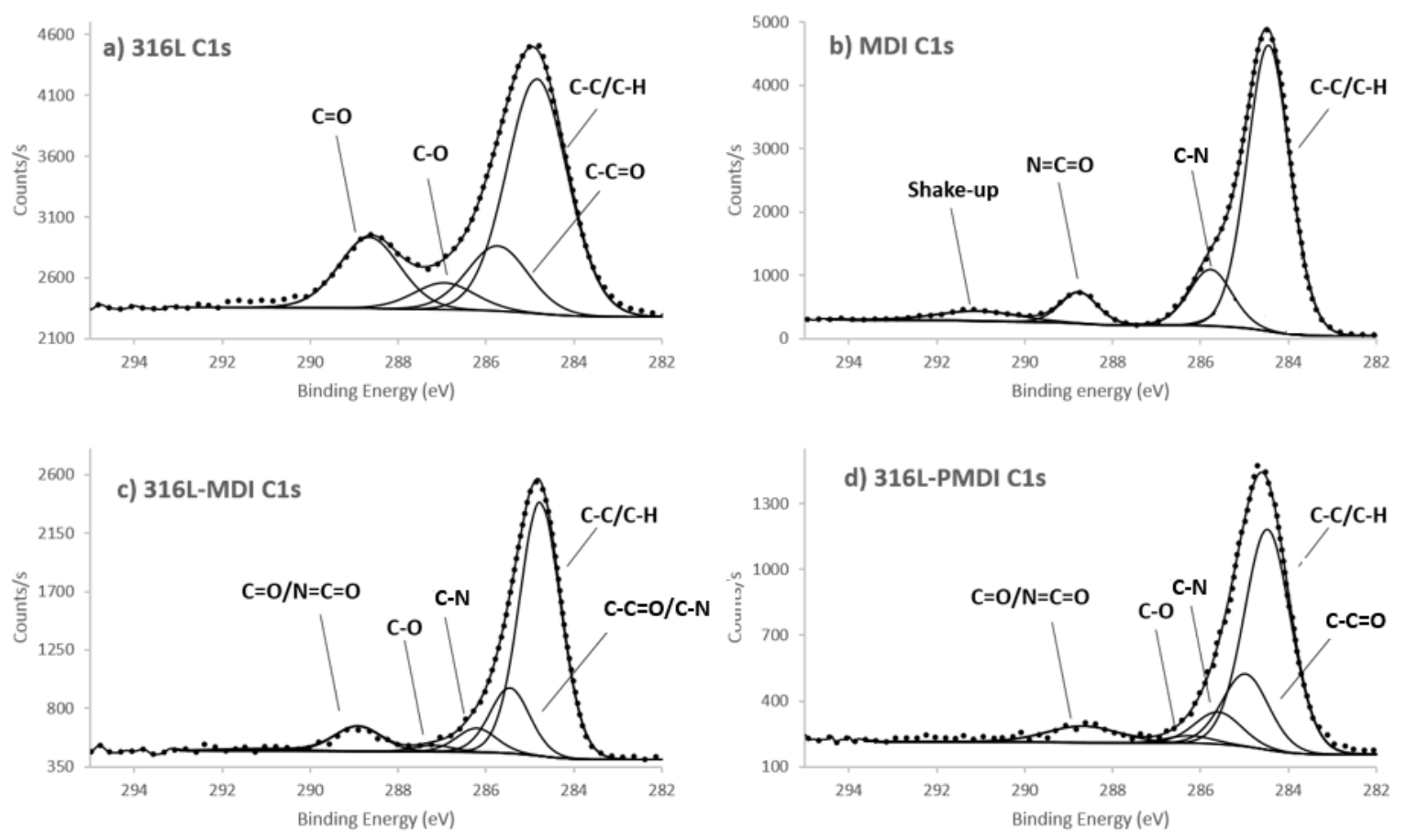

Figure 5: C1s high resolution spectra of a) oxidised 316L steel, b) MDI bulk, c) MDI-316L interface and d) PMDI 316L interface.
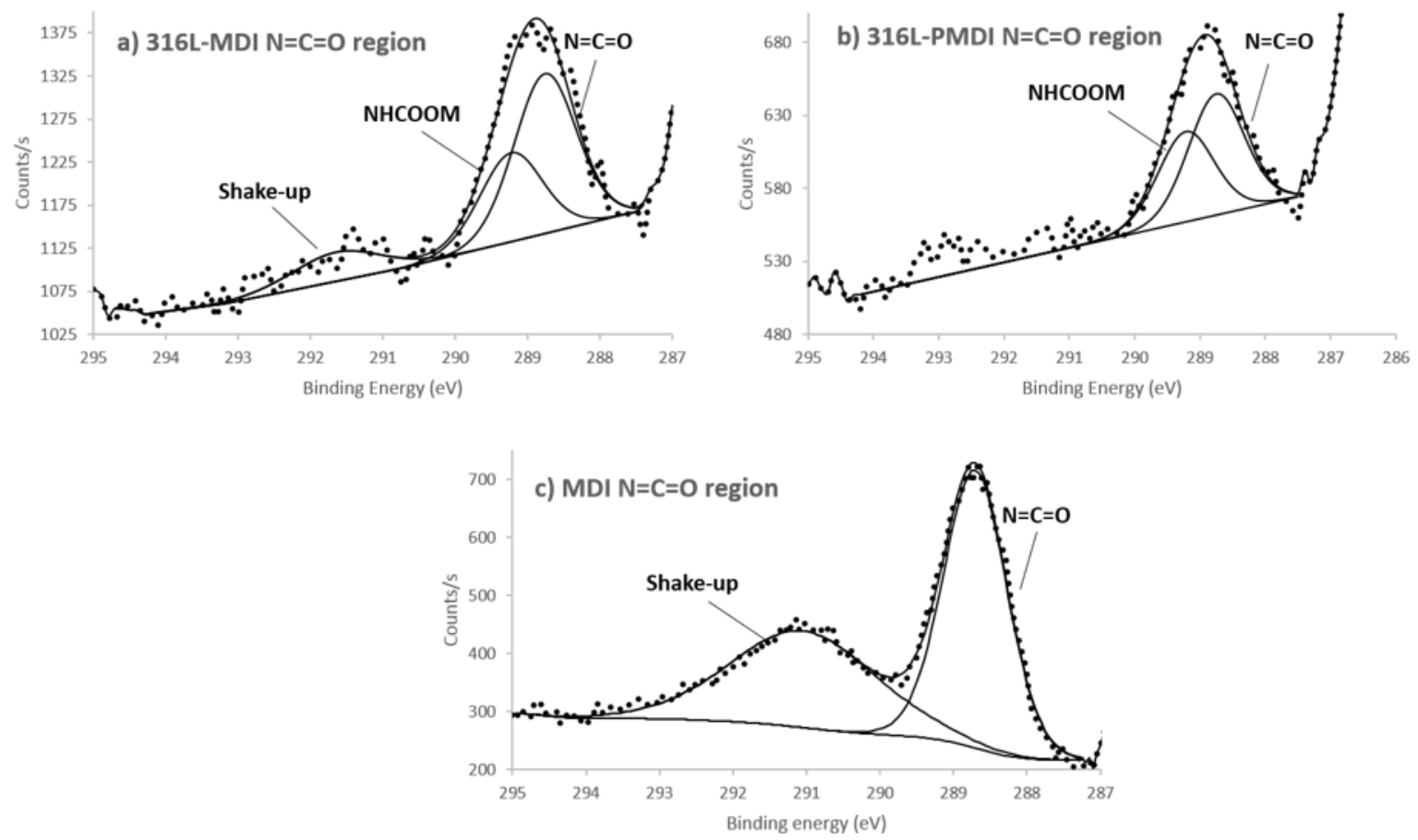

Figure 6: XPS C1s NCO region high resolution spectra of a) MDI-316L interface, b) PMDI-316L interface and c) MDI bulk. 

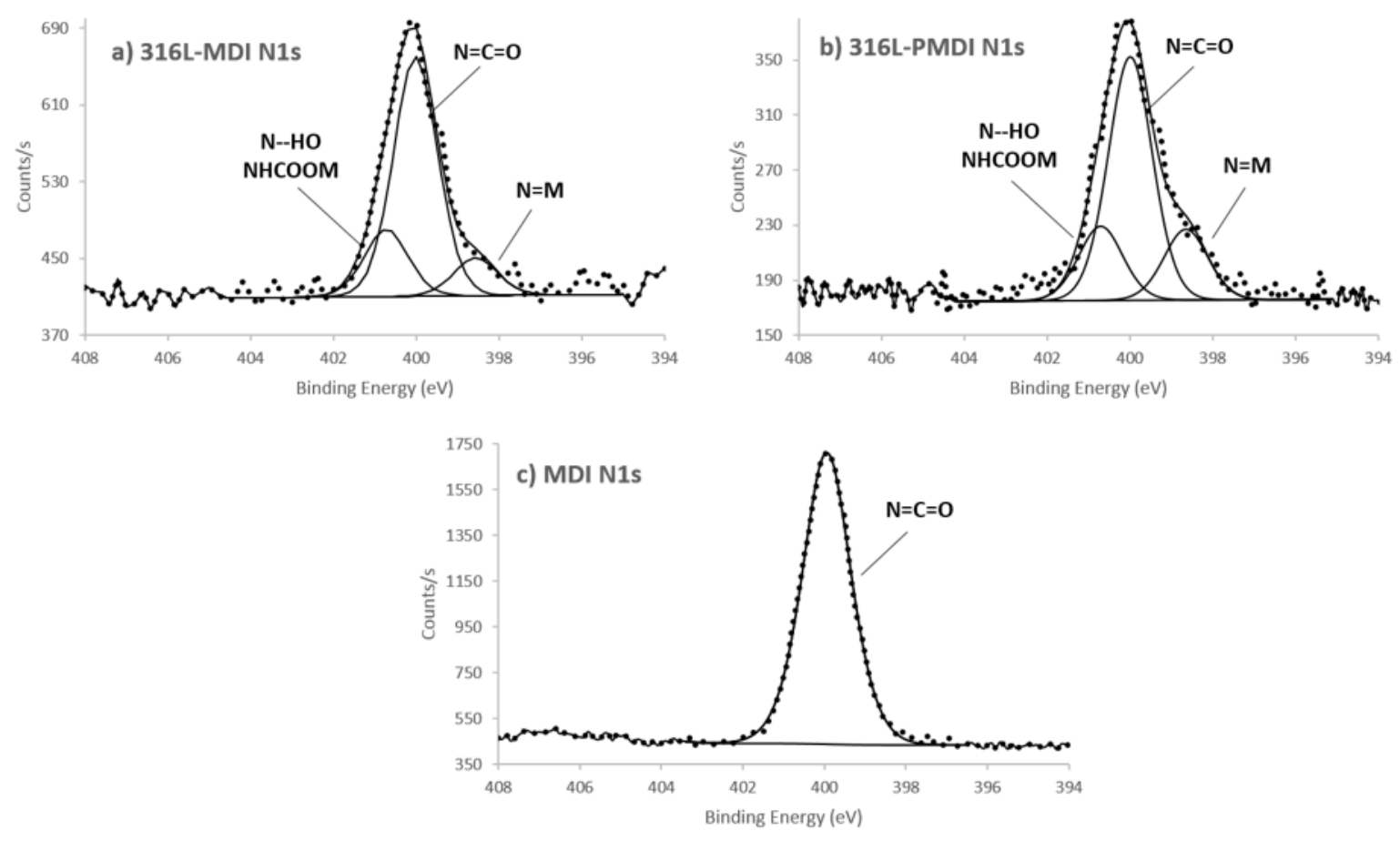

Figure 7: XPS N1s high resolution spectra of a) MDI-316L interface, b) PMDI-316L interface and c) MDI bulk.
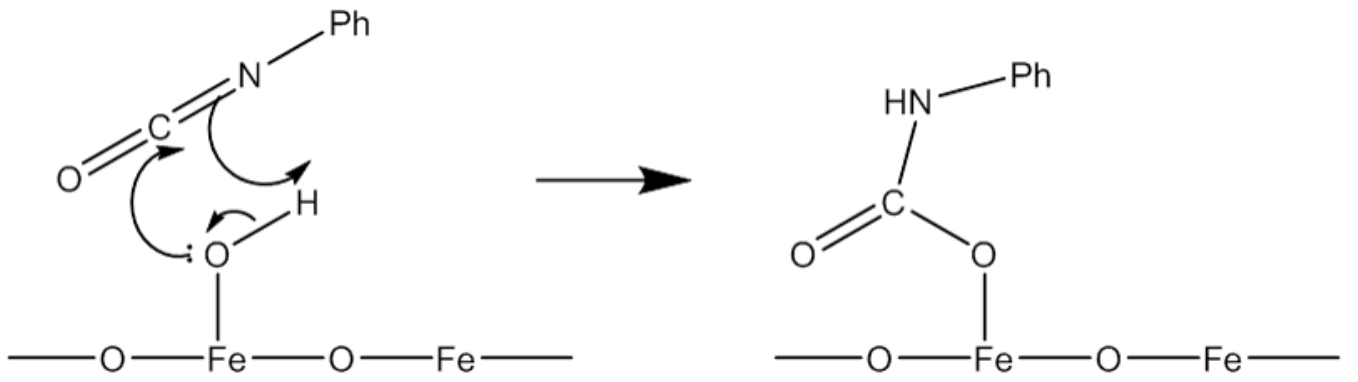

Figure 8: Schematic representation of the possible mechanism for the reaction of MDI with iron hydroxide.
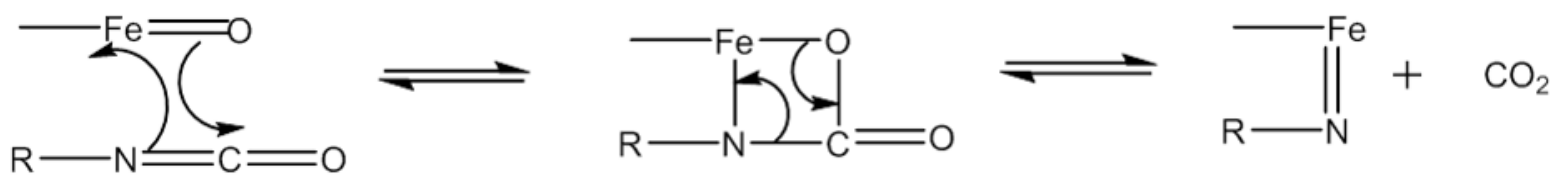

Figure 9: Schematic representation of the possible mechanism for the reaction between nitrogen in MDI and iron oxide. 
a)<smiles>CCCCCCOC(=O)Nc1ccc(C)cc1</smiles>

b)<smiles>Cc1ccc(N=CP)cc1</smiles>

Figure 10: Model molecules used for DFT binding energy prediction for the product of a) reaction between $\mathrm{MDI}$ and iron hydroxide and b) cycloaddition reaction of MDI to iron oxide. 

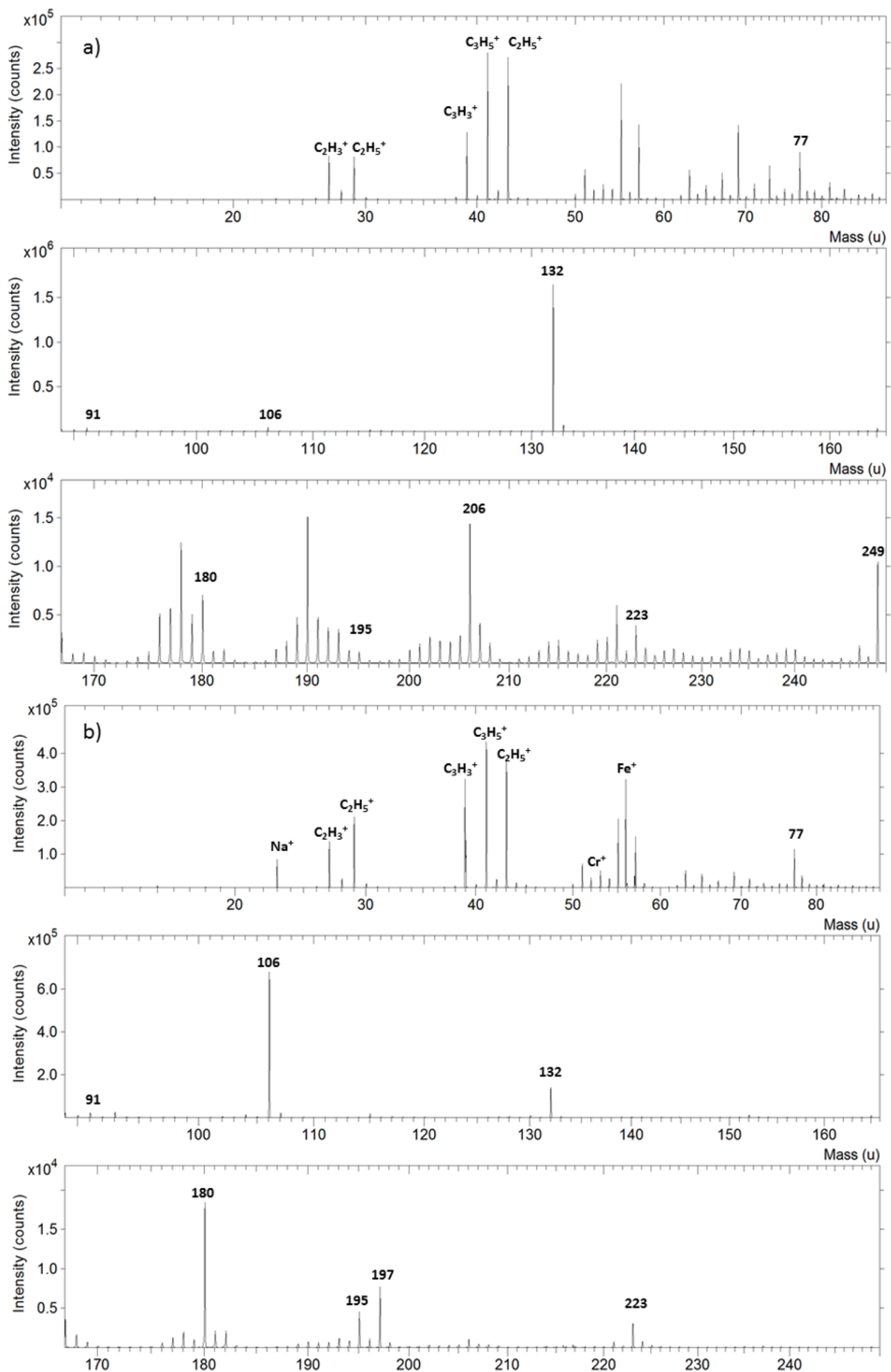

Figure 11: SIMS positive spectrum from 10 to 250 mass (u) of a) PMDI, b) PMDI-316L interface. 


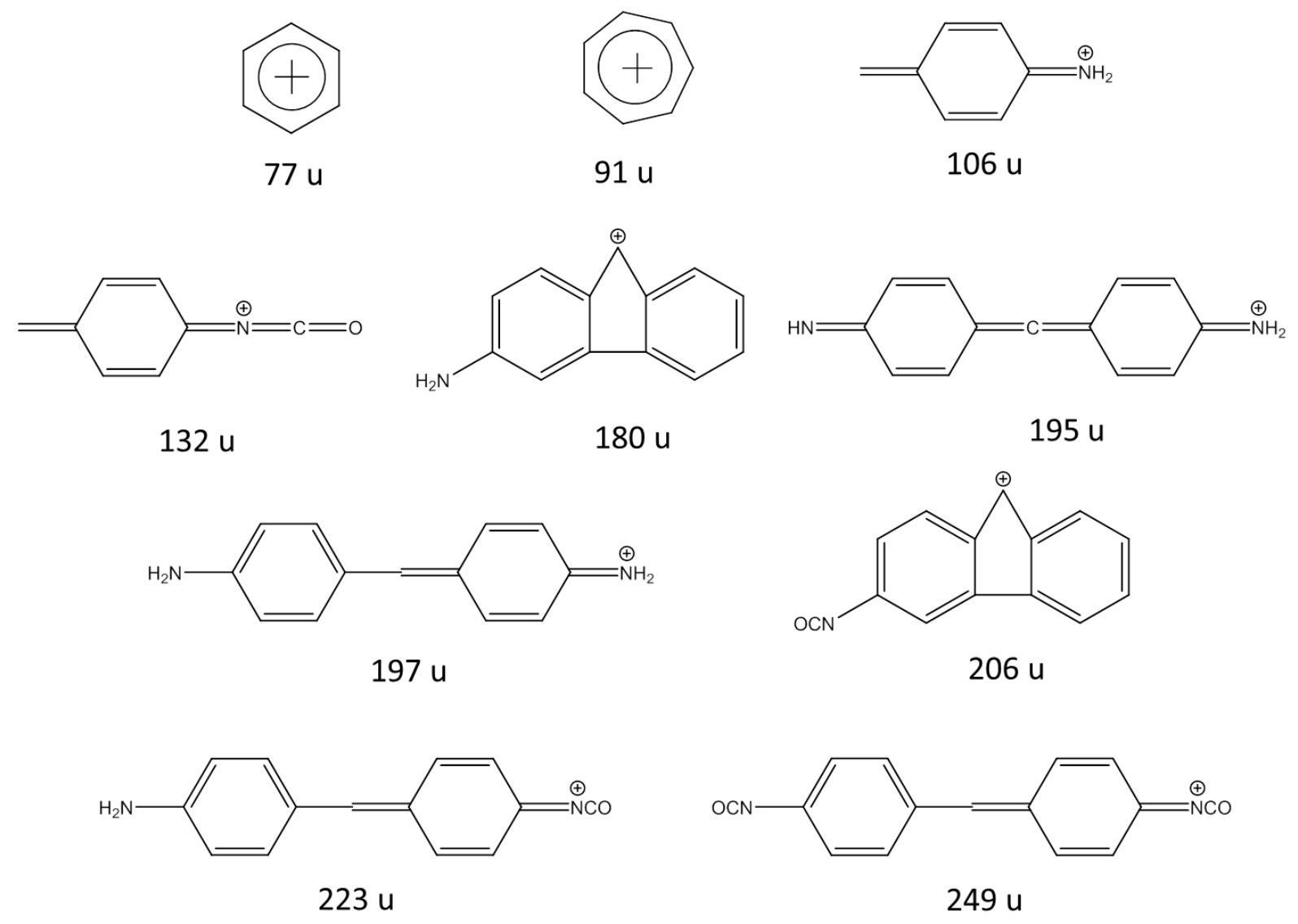

Figure 12: Possible structure of positive ion fragments from PMDI and relates molecules.

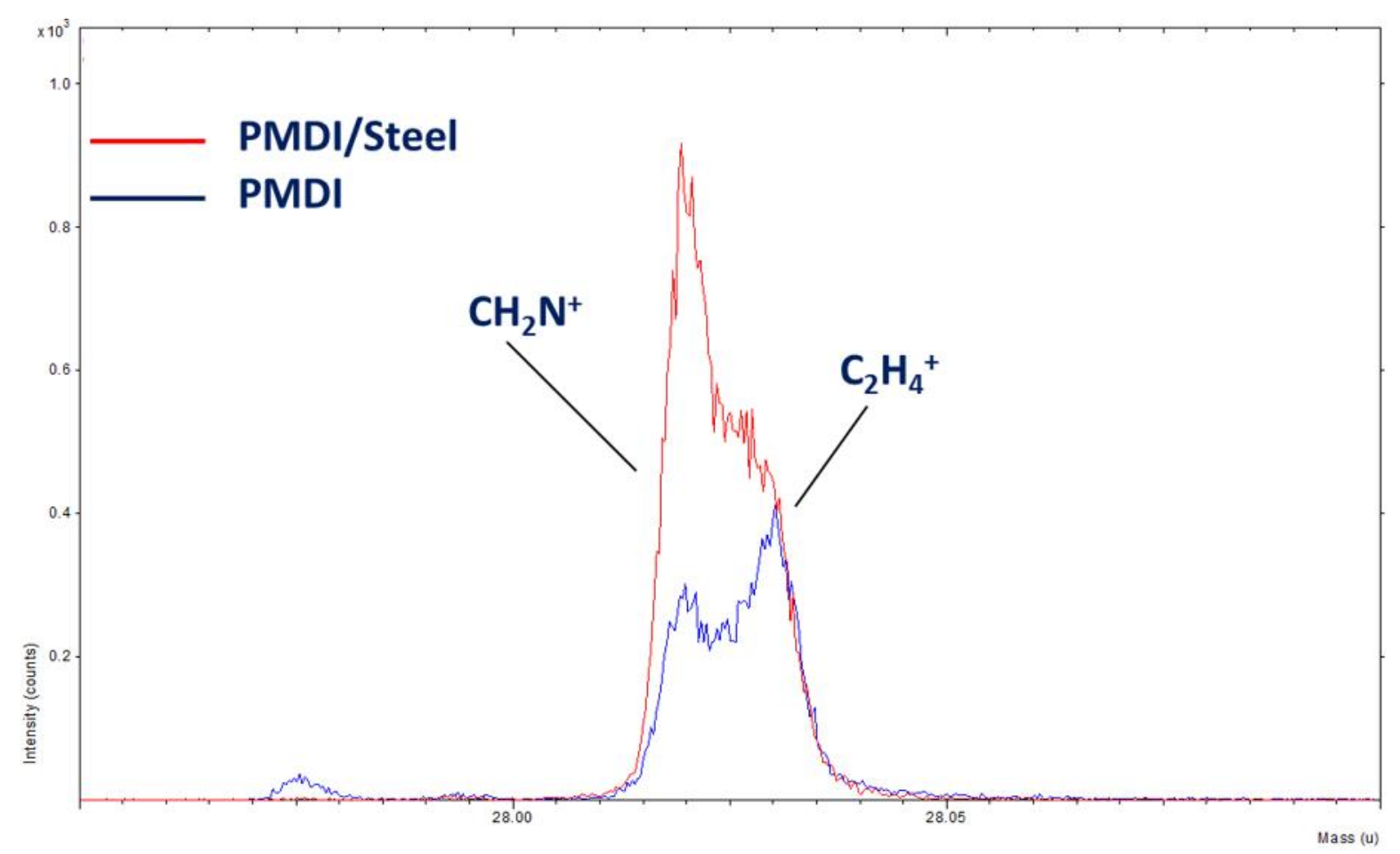

Figure 13: Pure PMDI and PMDI-316L interface peaks at $28 \mathrm{u}$. 


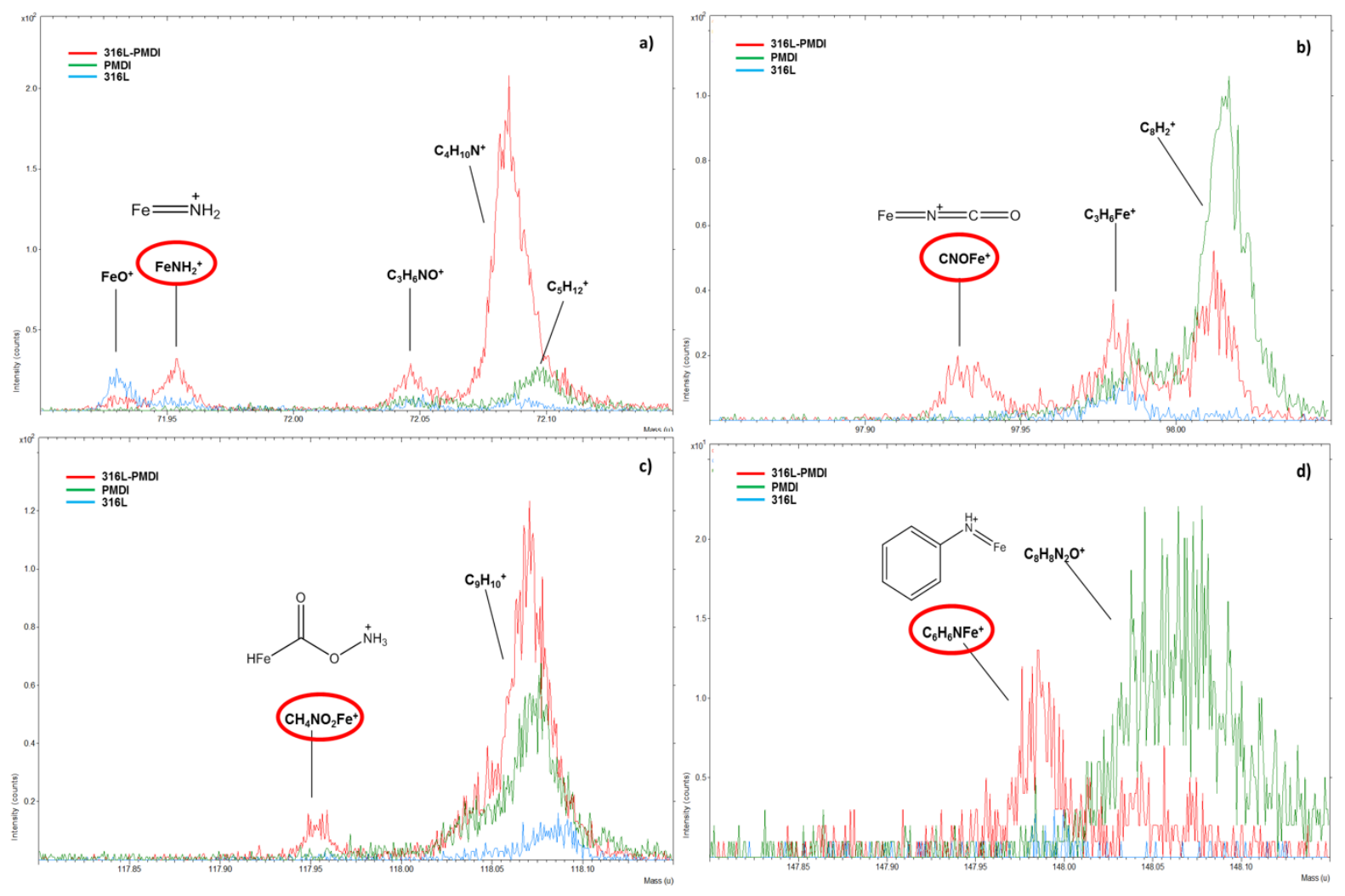

Figure 14: SIMS positive peaks around a) $72 u$, b) $98 u$, c) $118 u$ and d) $148 u$ nominal mass. 